\title{
MODELAGEM DE SÉRIES TEMPORAIS PARA DEMANDA E CONSUMO DE ENERGIA ELÉTRICA NO CAMPUS SANTA MÔNICA DA UNIVERSIDADE FEDERAL DE UBERLÂNDIA.
}

\author{
Marcelo José Pereira ${ }^{1}$ \\ Ednaldo Carvalho Guimarães ${ }^{2}$ \\ Jair Rocha do Prado³
}

RESUMO

Diante da relevância que a energia elétrica assume para as sociedades modernas e a necessidade de sua conscientização para o uso racional deste recurso, as Instituições de Ensino Superior (IES) representam uma importante instância, considerando tanto sua função formadora, quanto a aplicação de uma gestão voltada à eficiência energética. Neste sentido, o presente trabalho teve como objetivo caracterizar a utilização de energia elétrica no campus Santa Mônica da Universidade Federal de Uberlândia (UFU), tomando como base os registros de demanda de potência $(\mathrm{kW})$ e consumo de energia elétrica $(\mathrm{kWh})$ em horário de ponta $(\mathrm{HP})$ e horário fora de ponta (HFP), descrevendo seus componentes, e calculando previsões para períodos subsequentes através de modelos ajustados para as séries. A análise permitiu identificar, nas séries, a presença dos componentes, tendência e sazonalidade, associadas às questões estruturais para tendência e relacionadas aos calendários acadêmicos para a sazonalidade. Os modelos ajustados para todas as séries foi o Aditivo de Holt- Winters, que apresentaram um boa adequabilidade considerando os resultados obtidos para suas medidas de ajustes $\mathrm{R}^{2}$ e Erro de Porcentagem Absoluta Médio (MAPE) e para a validação de suas previsões. Esperase com este estudo, proporcionar apontamentos para a melhoria da gestão do uso de energia elétrica na UFU/Campus Santa Mônica, contribuindo para a eficiência energética e o uso racional dos recursos energéticos.

Palavras-chave: Instituições de ensino superior; Holt-Winters; Previsões.

\footnotetext{
${ }_{1}^{1}$ Mestre em Qualidade Ambiental pela Universidade Federal de Uberlândia (UFU).

2 Docente Faculdade de Matemática pela Universidade Federal de Uberlândia (FAMAT/UFU).

${ }^{3}$ Docente Faculdade de Matemática pela Universidade Federal de Uberlândia (FAMAT/UFU).
} 


\title{
TIMES SERIES MODELING FOR ELECTRICAL ENERGY DEMAND AND CONSUMPTION AT THE SANTA MÔNICA CAMPUS OF THE FEDERAL UNIVERSITY OF UBERLÂNDIA.
}

\begin{abstract}
In view of the relevance of electric energy to modern societies and the need to raise awareness about the rational use of this resource, Higher Education Institutions (HEls) represent an important instance, considering both its training function and the application of management focused on energy efficiency. In this sense, the objective of this work was to characterize the use of electric energy in the Santa Mônica campus of the Federal University of Uberlândia (UFU), based on the records of power demand $(\mathrm{kW})$ and electricity consumption (kWh) (HP) and off-peak time (HFP), describing its components, and calculating predictions for subsequent periods through seriesadjusted models. The analysis allowed to identify, in the series, the presence of the components, trend and seasonality, associated to the structural issues for trend and related to the academic calendars for the seasonality. The adjusted models for all series were the Holt-Winters Additive, which presented a good suitability considering the results obtained for their measures of adjustments $\mathrm{R}^{2}$ and Average Absolute Percent Error (MAPE) and for the validation of their predictions. The aim of this study is to provide guidelines for improving the management of electric energy use at UFU / Campus Santa Mônica, contributing to energy efficiency and the rational use of energy resources.
\end{abstract}

Keywords: Higher education institutions; Holt- Winters; Forecasts.

\section{INTRODUÇÃO}

A energia elétrica tem-se fundamentado como um importante recurso para a sustentação e desenvolvimento das sociedades modernas, se tornando gradualmente um bem de consumo essencial para todas as suas atividades. Com base neste cenário deve-se considerar a existência de uma conexão íntima entre energia renovável e a qualidade ambiental, acerca de sua geração (DINCER, 2000).

Neste sentido, o Brasil apresenta, ainda, condições favoráveis a uma trajetória sustentável para geração de energia elétrica, considerando a forte concentração em energias renováveis, principalmente a fonte hidráulica (GOLDEMBERG, 2015), aproximadamente 68\% (EPE, 2017). No entanto, a geração de energia elétrica 
nacional também representa danos ao meio ambiente, principalmente pelo processo de carbonização que a matriz energética brasileira tem enfrentado, com o aumento da participação das fontes de combustíveis fósseis, com a utilização crescente de termelétricas (GOLDEMBERG, 2015). Outro aspecto preocupante associado à concentração da energia gerada por hidrelétricas, é a sua dependência das variações climáticas e seus regimes hídricos (PEREIRA FILHO, 2015), além disso, essa fonte representa também danos ao meio ambiente, levando-se em conta, a necessidade de formação de grandes reservatórios, afetando assim, fauna e flora, aquática e terrestre, além das comunidades locais (MENKES, 2003 e SILVA e NASSAR, 2016).

Quanto à sua utilização, as Instituições de Ensino Superior (IES) apresentam uma função relevante, especialmente, no que tange ao uso racional e eficiente de energia elétrica (SILVA e NASSAR, 2016). Isso, representa a redução de sua utilização, consequentemente diminuição do desperdício e dos gastos, e retorno financeiro, concomitantemente numa perspectiva mais ampla, qualquer redução na demanda, ocasiona uma diminuição da sobrecarga para todo o sistema energético (SILVA e NASSAR, 2016). Prieto (2012) destaca que a utilização da energia elétrica é um importante indicador para avaliação do desempenho ambiental das universidades. Este recurso está associado a grandes impactos ambientais, além de representar grande parcela dos gastos destas instituições.

Pela perspectiva do uso da energia, torna-se relevante a necessidade das instituições/ organizações tanto do setor público quanto privado de estabelecer um plano de gestão, sendo que o seu gerenciamento requer o pleno conhecimento das normas que regulamentam a distribuição e o consumo da energia elétrica (ANEEL, 2010a e b), bem como os hábitos de utilização dessa determinada instituição ou instalação (OLIVEIRA, 2006).

O principal objetivo do estudo de séries temporais é identificar estatisticamente um modelo, que apresenta o melhor ajuste para as séries históricas a serem analisadas, considerando seus registros, no caso deste estudo, do consumo e demanda de energia elétrica na UFU, incluindo também o cálculo de seus valores futuros. Tem-se como hipóteses que a análise da energia elétrica e o comportamento do consumo e demanda de potência podem ser descritos por modelos de séries 
temporais. Esses modelos terão potencial para balizar decisões administrativas institucionais que permitam uma racionalização e melhor uso da energia elétrica, pois espera-se evidenciar claramente sazonalidade e tendência na utilização de energia elétrica na UFU/ Santa Mônica.

Assim sendo, este estudo propõe caracterizar os hábitos, de consumo de energia elétrica, para o campus Santa Mônica da Universidade Federal de Uberlândia (UFU), por meio das séries temporais para as variáveis de demanda de potência e consumo de energia em horário de ponta (HP) e em horário fora de ponta (HFP).

\section{MATERIAL E MÉTODOS}

Os dados avaliados para o consumo de energia elétrica, no Campus Santa Mônica, da UFU, em Uberlândia-MG, foram obtidos por meio das faturas emitidas pela Companhia Energética de Minas Gerais (CEMIG).

A cidade de Uberlândia localiza-se na região central do Brasil, no Estado de Minas Gerais, na mesoregião do Triângulo Mineiro e Alto Paranaíba. A UFU possui sete campi, quatro deles localizados no município de Uberlândia (MG), um em Ituiutaba (MG), um em Monte Carmelo (MG) e um em Patos de Minas (MG). Uberlândia se encontra na latitude de $18^{\circ} 55^{\prime}$ 25'S, longitude de 48 17' 19' W. Segundo a classificação de Köppen, o clima da região é do tipo Aw, isto é, tropical quente úmido com inverno frio e seco.

Em 2017, a Universidade manteve um total de 26.855 alunos matriculados, tanto na graduação quanto na pós-graduação (mestrado e doutorado) e 4.904 servidores, entre técnicos e docentes, distribuídos em uma área construída total de $359.695 \mathrm{~m}^{2}$. Deste total de usuários, refere-se ao campus Santa Mônica, aproximadamente 15.800 alunos e 2.700 servidores desenvolvendo suas atividades, totalizando cerca de 18.500 usuários, numa área construída total de $117.295 \mathrm{~m}^{2}$ (PROPLAD/DIRPL/DIESI/ UFU, 2017) e (CAPES, 2017).

As variáveis analisadas neste estudo foram: i) demanda de potência e suas variações em HP e em HFP; e ii) consumo de energia elétrica e suas variações em HP e em HFP, no período de janeiro de 2004 a dezembro de 2017, totalizando, assim, 
168 observações para cada série. Ressalta-se que, entre os registros apresentados, obteve-se 154 observações válidas, considerando algumas lacunas encontradas, entre elas, o ano de 2006 e os meses de novembro e dezembro de 2007, períodos sem registros não tendo sido possível a localização dos documentos físicos (faturas), tão pouco registros históricos em sistemas de controle, em parte pela ausência de registros eletrônicos da UFU e também por conta da temporalidade de arquivamento deste tipo de documentos (CONARQ, 2001). Os valores referentes ao ano de 2018 foram utilizados para comparação com as previsões obtidas, como medida de validação dos modelos ajustados.

Inicialmente, a série foi analisada graficamente (SILVA, et al., 2008), e considerando as características identificadas visualmente, tais como a tendência e a sazonalidade, e com base no comportamento geral das séries foi possível deduzir que o comportamento de seus dados seguem um padrão aditivo (BRILLINGER, 2015). De acordo com Morettin e Toloi (2006) e Ferraz (1999), testou-se a tendência e a sazonalidade pelo Teste $\mathrm{F}$ (ANOVA). A partir deste ponto, buscou-se identificar, por meio do software IBM SPSS Statistics $20 \AA$ (SPSS), e sua aplicação "expert modeler" (KHALIQ, et. al. 2015), o modelo que melhor explica o comportamento das séries, considerando seus parâmetros tais como o nível, tendência e sazonalidade, analisando também os critérios para explicação dos resultados obtidos pelo modelo, considerando, a significância em $5 \%$, para os valores do $\mathrm{R}^{2}$, do Erro de porcentagem absoluta média (MAPE), da autocorrelação dos resíduos e validação de suas previsões para o ano de 2018.

Visando justificar possíveis ocorrências externas no comportamento da demanda e consumo de energia elétrica, verificou-se a contribuição do Programa de Apoio a Planos de Reestruturação e Expansão das Universidades Federais (REUNI) para tendência de crescimento tanto da demanda como do consumo de energia elétrica, realizou-se uma análise histórica do desenvolvimento da UFU, com enfoque para o Campus Santa Mônica, considerando o aumento do número de usuários do campus (estudantes e servidores) e também o incremento da área construída $\left(\mathrm{m}^{2}\right)$, o levantamento foi pautado no REUNI a partir do Decreto no 6.096 , de 24 de abril de 2007(Presidência da República), e também no Plano de Expansão da UFU, 2008- 
2012. Examinou-se também as resoluções dos Conselhos Superiores da UFU, afim de apurar os períodos de férias e de atividades letivas.

\section{RESULTADOS E DISCUSSÕES}

\subsection{ANÁLISE EXPLORATÓRIA DAS SÉRIES DE DEMANDA E CONSUMO DE ENERGIA ELÉTRICA}

A análise estatística exploratória possibilitou a identificação visual de características importantes do comportamento da utilização de energia elétrica no campus Santa Mônica da UFU, destaca-se entre elas os indícios de tendência e sazonalidade, fundamentais para a análise de séries temporais e a identificação de seus modelos. Estas características foram identificadas também por Prado et. al. (2014) em suas análises acerca da demanda de potência na Universidade Federal de Lavras (UFLA). Outros trabalhos, em áreas distintas, também identificaram, visualmente, características de tendência e/ou sazonalidade, em suas séries, como a análise da precipitação de chuva feita por Puah et. al (2016) e o estudo sobre uma série de registros de temperatura climática em Uberlândia, MG, realizada por Silva et. al (2008), sendo nestes casos identificadas caracteríticas sazonais apenas.

A Figura 1 apresenta o comportamento da demanda de potência HP e HFP no campus Santa Mônica da UFU, considerando seus registros mensais, ao longo do período analisado. Pode-se perceber a presença de indícios de sazonalidade, considerando os picos recorrentes ao longo da série, também nota-se a presença de tendência, tendo em vista a inclinação significativa na análise completa dos registros da série. 


\section{sunisul}

Figura 1 - Série mensal de demanda de potência medida HP e HFP (kW) na UFU/ Campus Santa Mônica, no período de jan/04 a dez/17.

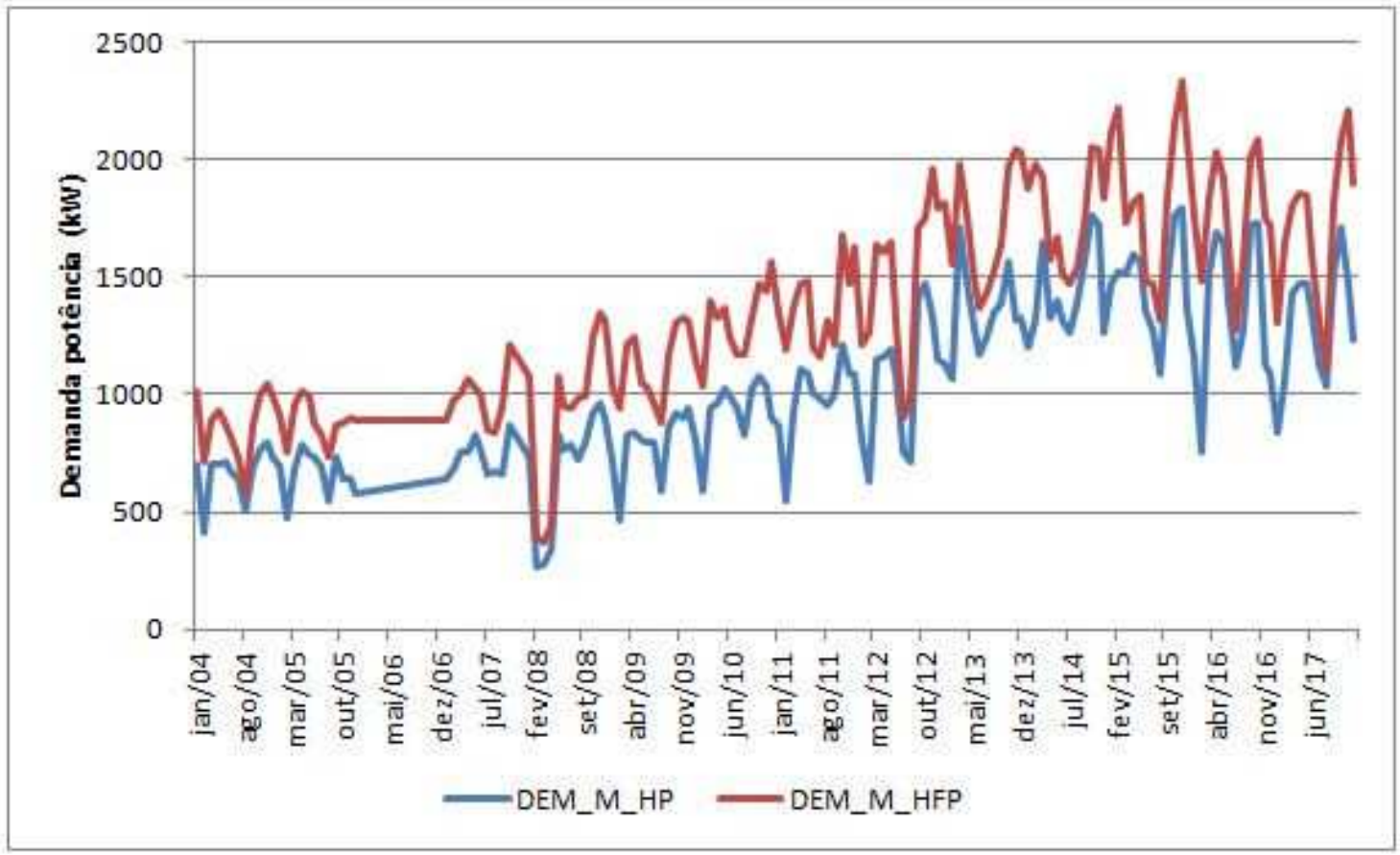

Fonte: Autor, 2018.

A Figura 2 apresenta o comportamento do consumo de energia elétrica HP e HFP no campus Santa Mônica da UFU, considerando seus registros mensais, ao longo do período analisado. Percebe-se a presença de características de sazonalidade, considerando a variação regular nos dados em subperíodos recorrentes ao longo da série, também nota-se a presença de tendência, tendo em vista o movimento persistente nos dados em uma dada direção da série. 


\section{¿UNISUl}

Figura 2 - Série mensal de consumo de energia (kWh) HP e HFP na UFU/ Campus Santa Mônica, no período de jan/04 a dez/17.

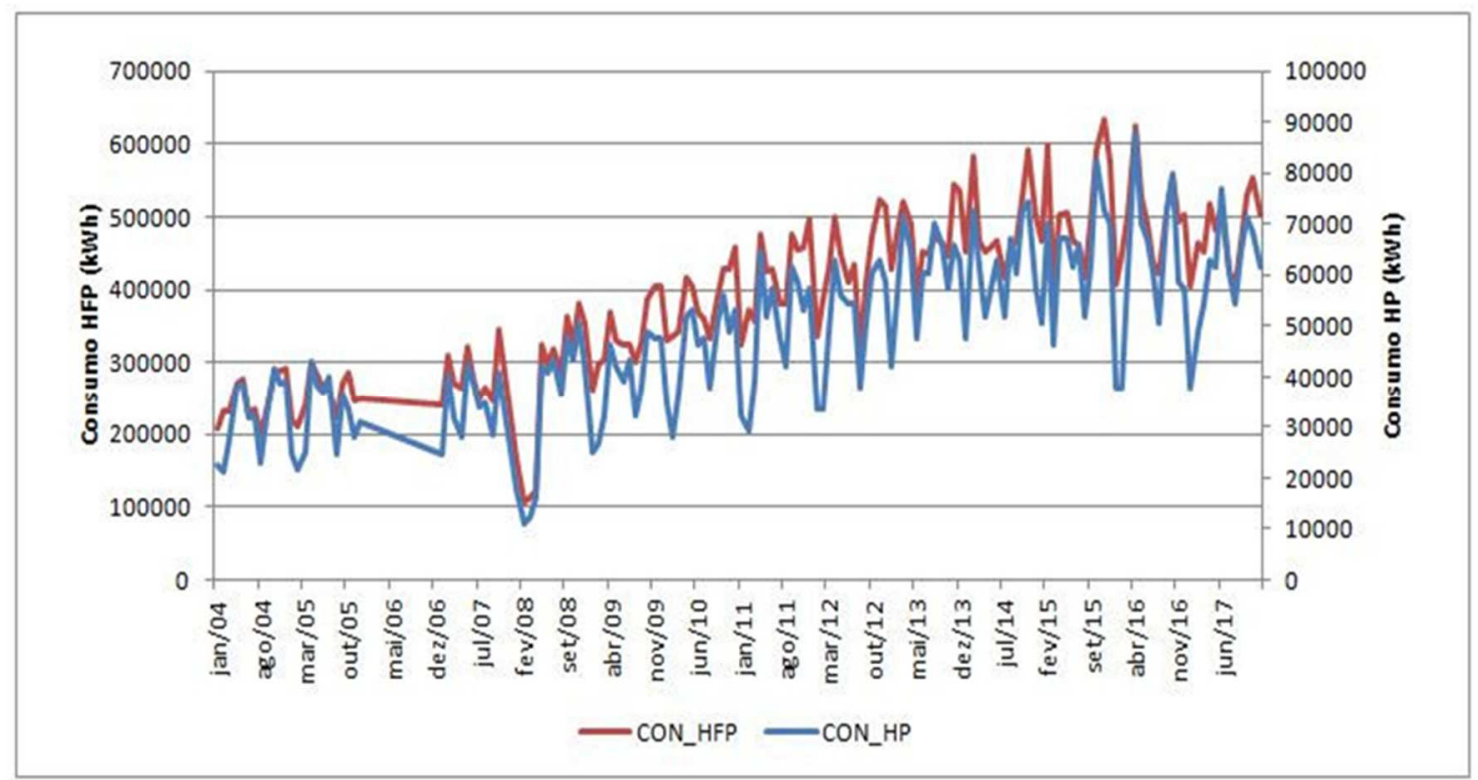

Fonte: Autores, 2018.

As Figuras 1 e 2 apresentam indícios de tendência de crescimento, ao longo da série, da demanda e do consumo de energia elétrica no campus Santa Mônica da UFU, tanto para HP como para HFP. Acredita-se que, em parte, esse crescimento tenha sido ocasionado pelo desenvolvimento e expansão do referido campus da UFU, considerando as políticas de investimentos do Governo Federal, principalmente no decênio de 2004 à 2013, com o REUNI e outros programas de expansão das universidades públicas brasileiras, o que representou um incremento em área construída, aquisição de equipamentos e aumento do número de usuários por meio da abertura de novas vagas para estudantes, docentes e técnicos administrativos, decorrentes da abertura de novos cursos, novas turmas e novos turnos de cursos já existentes. 


\section{GESTÃO \& SUSTENTABILIDADE} AMBIENTAL

\section{Unisul}

$\mathrm{Na}$ Tabela 1 é apresentado o crescimento acumulado médio para demanda de $95,61 \%$ e para o consumo de $89,9 \%$, destacando os registros iniciais no ano 2004 e finais em 2017, para as variáveis de demanda e consumo HP e HFP, corroborando com os indícios de tendência identificados visualmente nas séries, tendo sido possível notar um crescimento mais acentuado a partir do ano de 2008, com o pico de maior demanda e consumo no ano de 2015.

Tabela 1 - Crescimento da demanda e consumo HP e HPF, considerando a média anual, no Campus Santa Mônica da UFU.

\begin{tabular}{cccccc}
\hline & & $\begin{array}{c}\text { Inicial - } \\
\mathbf{2 0 0 4}\end{array}$ & $\begin{array}{c}\text { Final - } \\
\mathbf{2 0 1 7}\end{array}$ & $\begin{array}{c}\text { Crescimento } \\
\text { acumulado }\end{array}$ & $\begin{array}{c}\text { Média de } \\
\text { crescimento }\end{array}$ \\
\hline $\begin{array}{c}\text { Demanda } \\
\text { medida (kW) }\end{array}$ & HP & 669,58 & 1.309 & $95,49 \%$ & \\
\hline $\begin{array}{c}\text { Consumo } \\
\text { (kWh) }\end{array}$ & HP & $32.153,33$ & $60.316,67$ & $87,59 \%$ & \\
& HFP & $247.566,7$ & $475.883,3$ & $92,22 \%$ & $89,9 \%$ \\
\hline
\end{tabular}

Fonte: Autores, 2018.

Para a fundamentação da análise considerando os indícios de tendência de crescimento da demanda e consumo de energia elétrica na UFU, e o aporte do REUNI, buscou-se levantar as informações referentes ao número de usuários e da área construída no campus Santa Mônica da UFU, no período correspondente à série temporal em questão. Neste sentido apurou-se um crescimento acumulado de aproximadamente $75,85 \%$ do número de usuários (Tabela 2 e Tabela 4), representando um crescimento médio anual de 5,47\%, considerando o número de discentes e servidores (docentes e técnicos administrativos) e uma expansão de $52,25 \%$ da área construída, representando um crescimento médio anual de 3,73\%. (Tabela 3 e Tabela 4). (PROPLAD/DIRPL/DIESI/UFU, 2017), (CAPES, 2017) e (PRIETO, 2005). 


\section{sunisul sim}

Tabela 2 - Evolução do número de usuários do campus Santa Mônica da UFU, considerando as categorias discente e servidores (docentes e técnicos administrativos).

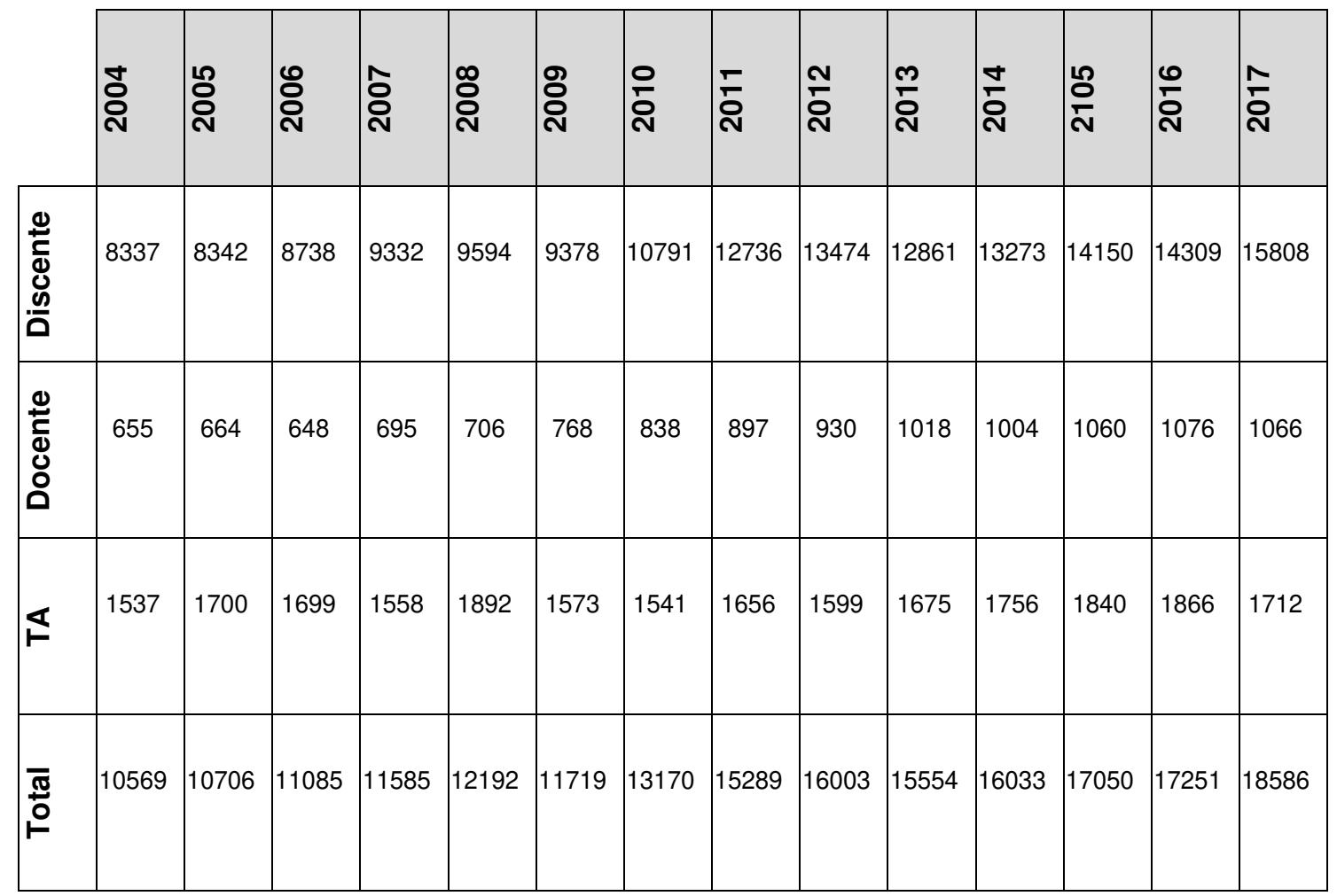

Fonte: PROPLAD/DIRPL/DIESI/ UFU e CAPES, 2017.

Tabela 3 - Evolução da área construída na UFU/ Santa Mônica $\left(\mathrm{m}^{2}\right)$.

\begin{tabular}{|c|c|c|c|c|c|c|c|}
\hline \multirow{4}{*}{ 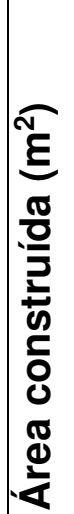 } & 2004 & 2005 & 2006 & 2007 & 2008 & 2009 & 2010 \\
\hline & 77034,84 & $\begin{array}{l}\text { Não } \\
\text { identificado }\end{array}$ & $\begin{array}{l}\text { Não } \\
\text { identificado }\end{array}$ & $\begin{array}{l}\text { Não } \\
\text { identificado }\end{array}$ & $\begin{array}{l}\text { Não } \\
\text { identificado }\end{array}$ & 91783,46 & 91783,46 \\
\hline & 2011 & 2012 & 2013 & 2014 & 2105 & 2016 & 2017 \\
\hline & 102811,46 & 102811,46 & 110100,46 & 110100,46 & 116144,00 & 117295,01 & 117295,01 \\
\hline
\end{tabular}




\section{GESTÃO \& SUSTENTABILIDADE AMBIENTAL \\ ¿unisul}

Fonte: PRIETO, 2005 e PROPLAD/DIRPL/DIESI/ UFU, 2017.

Tabela 4 - Expansão da UFU/ Santa Mônica, número de usuários e área construída.

\begin{tabular}{lcccc}
\hline & $\begin{array}{c}\text { Inicial - } \\
2004\end{array}$ & $\begin{array}{c}\text { Final - } \\
\mathbf{2 0 1 7}\end{array}$ & Crescimento & $\begin{array}{c}\text { Média de } \\
\text { crescimento } \\
\text { anual }\end{array}$ \\
\hline Número de usuários & 10.569 & 18.586 & $75,85 \%$ & $5,41 \%$ \\
\hline Área construída $\left(\mathbf{m}^{2}\right)$ & $77.034,84$ & $117.295,01$ & $52,25 \%$ & $3,73 \%$ \\
\hline
\end{tabular}

Fonte: Autores, 2018.

Conforme observado por Lo (2013), em seu estudo acerca do comportamento do uso de energia elétrica nas IES chinesas, o consumo e a demanda de energia elétrica nestas instituições podem apresentar variações, considerando seus aspectos estruturais, como a área construída e o número de usuários. Serna et. al (2011), consideram a expansão, que as IES brasileiras vivenciaram principalmente a partir do ano de 2008, como um fator preponderante para a tendência de crescimento do consumo e da demanda de energia elétrica. Ratificando, assim, a importância da caracterísitica, de tendência, identficada na análise acerca da contribuição da expansão da UFU/Santa Mônica, no período analisado. Prado et. al. (2014) percebeu movimento similar também em suas observações referentes ao comportamento da utilização de energia elétrica na UFLA.

Outra característica identificada na análise refere-se à sazonalidade, considerando o comportamento geral da série, para demanda e consumo HP e HFP, sendo que o mesmo demonstra uma forte relação com os períodos definidos pelos calendários acadêmicos, sendo possível evidenciar os períodos de férias e os semestres letivos, neste último com picos de utilização de energia principalmente em seus encerramentos, reforçando, assim, as características da variação sazonal.

Com base na inspeção visual dos gráficos gerados para a análise preliminar, de acordo com Morettin e Toloi (2006) e considerando as características identificadas de tendência e sazonalidade, sendo o padrão observado, para sazonalidade, 


\section{GESTÃO \& SUSTENTABILIDADE AMBIENTAL}

\section{¿Unisul}

estacionário de amplitude, tem-se apontamento para séries com propriedades que permitem classificá-las como sendo aditivas. Neste sentido, buscou-se aplicar também o teste de decomposição sazonal, este procedimento, decompõe a série em um componente sazonal, com o intuito de averiguar o componente de sazonalidade, contribuindo para a identificação do comportamento da série, e sua característica aditiva. Na Tabela 5, são apresentados os valores da decomposição sazonal para as variáveis de demanda e consumo HP e HFP.

Tabela 5 - Valores para decomposição sazonal, modelo aditivo, demanda e consumo HP e HFP, na UFU/ Santa Mônica.

\begin{tabular}{|c|c|c|c|c|}
\hline & $\begin{array}{c}\text { DEMANDA_HP } \\
(\mathrm{kW})\end{array}$ & $\begin{array}{c}\text { DEMANDA_MEDIDA } \\
\text { _HFP (kW) }\end{array}$ & $\begin{array}{c}\text { CONSUMO_HP } \\
\text { (kWh) }\end{array}$ & $\begin{array}{c}\text { CONSUMO_HFP } \\
\text { (kWh) }\end{array}$ \\
\hline Período & Fator Sazonal & Fator Sazonal & Fator Sazonal & Fator Sazonal \\
\hline 1 & $-119,179$ & $-6,916$ & $-14368,028$ & $-56806,269$ \\
\hline 2 & $-263,790$ & $-97,756$ & $-8913,861$ & $-11885,714$ \\
\hline 3 & 64,849 & 50,355 & $-5493,583$ & $-29241,825$ \\
\hline 4 & 73,168 & 41,619 & 4874,195 & 21836,786 \\
\hline 5 & 96,467 & 50,049 & 3172,806 & 8515,397 \\
\hline 6 & $-4,762$ & $-141,458$ & 4061,417 & $-5329,047$ \\
\hline 7 & $-93,192$ & $-259,221$ & 1016,692 & $-25050,955$ \\
\hline 8 & $-130,908$ & $-226,881$ & $-5077,472$ & $-46084,603$ \\
\hline 9 & 92,495 & 6,750 & 2826,695 & 692,897 \\
\hline 10 & 200,856 & 206,639 & 8541,417 & 36812,897 \\
\hline 11 & 104,759 & 212,750 & 6117,861 & 60046,481 \\
\hline 12 & $-20,762$ & 164,070 & 3241,861 & 46493,953 \\
\hline
\end{tabular}

Fonte: Autores, 2018.

A decomposição sazonal, em seu modelo aditivo, apresenta a variação em torno das médias mensais, destacam-se os meses com registros abaixo da média, comum a todas as variáveis, sendo: i) janeiro; ii) fevereiro e; iii) agosto. Os meses com registro de ultrapassagem: i) abril; ii) maio; iii) setembro; iv) outubro e; v) novembro. Essa variação apresentada corrobora com a relação 


\section{GESTÃO \& SUSTENTABILIDADE AMBIENTAL \\ unisul}

identificada para o comportamento da utilização de energia elétrica tendo como fator principal os períodos letivos e de férias definidos pelos calendários acadêmicos da UFU.

Identificou-se por meio das resoluções dos Conselhos Superiores da UFU, que estabeleceram os calendários acadêmicos do período analisado, que habitualmente os meses de férias compreendem: no início do ano, janeiro, fevereiro e meados de março e entre o primeiro e segundo semestres, se concentram em meados de julho e meados de agosto principalmente.

Os demais meses que apresentam comportamento da variação acima da média representam respectivamente os meses letivos dos primeiro e segundo semestres, compreendendo o pleno funcionamento das instalações do campus Santa Mônica da UFU com a presença efetiva de seus usuários, estudantes e servidores, desenvolvendo suas atividades de forma plena. Possíveis variações encontradas no comportamento sazonal das séries analisadas podem estar associadas à períodos de greves e ou reposições de aulas, bem como outros eventos ocorridos de ordens diversas, como por exemplo: o adiamento do primeiro semestre de 2009 decorrentes do surto de gripe H1N1, ou a modificação no período de férias no ano de 2014 por conta da realização da copa do mundo de futebol no Brasil. Silva e Nassar (2016) notaram comportamento semelhante no Instituto Federal Fluminense (IFF), com relação a dimunição do uso de energia elétrica nos períodos de férias, recessos de fim de ano, carnaval e período de greves, o que representa a ausência de grande parte dos usuários do campus.

\subsection{AJUSTE DOS MODELOS DE SÉRIES TEMPORAIS PARA DEMANDA DE POTÊNCIA E CONSUMO DE ENERGIA ELÉTRICA.}

Considerando as características, identificadas visualmente, por meio da análise estatística exploratória, como a tendência, e a sazonalidade, estas são confirmadas pelos resultados do Teste de ANOVA, para as variáveis, consumo e demanda HP e HFP. Para o fator ano, obteve-se p-valor < 0,001, portanto altamente significativo, ratificando assim, a presença de tendência. Para o fator 


\section{GESTÃO \& SUSTENTABILIDADE} AMBIENTAL

\section{unisul sims}

mês, identificou-se p-valor $<0,001$, altamente significativo, adimitindo sazonalidade na série. Ferraz (1999), em sua análise sobre séries de precipitações de chuva em Lavras, utilizando-se do teste ANOVA, obteve resultado similar para o fator mês, indicando sazonalidade na série observada, e para o fator ano, o autor encontrou p-valor não significativo, indicando assim, uma série sem tendência.

Com base no comportamento dos dados referentes à demanda e o consumo, comprova-se por meio desses indícios que o método de suavização exponencial e seu modelo aditivo de Holt-Winters se enquadra de maneira satisfatória para a explicação e previsão da série, conforme resultado obtido na análise estatística, por meio do SPSS. Esse mesmo modelo mostrou-se adequado também para o ajuste de séries temporais de chuvas, utilizado para examinar o padrão de precipitação na bacia do Rio Langat, Malasia, conforme apresentado por Puah et. al (2016).

A partir da identificação do modelo aditivo de Holt-Winters para todas as variáveis, foram calculados seus parâmetros de suavização exponencial sendo o nível, a tendência e a sazonalidade, considerando os valores obtidos para a estimativa. Todos os parâmetros obtidos foram listados na Tabela 6 .

Tabela 6 - Parâmetros do modelo de suavização exponencial, considerando a estimativa.

\begin{tabular}{llll}
\hline Variável & Nível & Tendência & Sazonalidade \\
\hline Demanda HP (kW) & 0,277 & 0,000393 & 0,001000 \\
Demanda HFP (kW) & 0,602 & 0,000011 & 0,000175 \\
Consumo HP (kWh) & 0,140 & 0,001171 & 0,001000 \\
Consumo HFP (kWh) & 0,302 & 0,000011 & 0,001000 \\
\hline
\end{tabular}

Fonte: Autores, 2018.

A adequação do modelo obtido foi inferida por $R^{2}$ e MAPE, considerando que: i) $R^{2}$ é o coeficiente de determinação, sendo a proporção de variação na 


\section{GESTÃO \& SUSTENTABILIDADE} AMBIENTAL

\section{¿unisul sim}

variável dependente explicada pelo modelo de regressão e; ii) MAPE, medida de quanto uma série dependente varia de seu nível previsto pelo modelo. Seus resultados para todo o período do estudo foram registrados na Tabela 7.

Tabela 7 - Valores de adequação dos modelos para utilização de energia elétrica

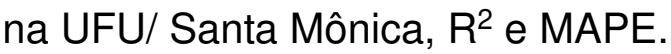

\begin{tabular}{lll}
\hline Variável & $\mathbf{R}^{\mathbf{2}}$ & MAPE (\%) \\
\hline Demanda - HP (kW) & 0,830 & 12,990 \\
Demanda - HFP (kW) & 0,835 & 10,963 \\
Consumo - HP (kWh) & 0,772 & 13,809 \\
Consumo - HFP (kWh) & 0,849 & 9,796 \\
\hline
\end{tabular}

Fonte: Autores, 2018.

Com base nos resultados obtidos pelo $\mathrm{R}^{2} \mathrm{o}$ ajuste do modelo para a demanda tanto em HP quanto em HFP, podem ser explicado em 83\% dos casos. Para a variável consumo observa-se uma diferença maior na precisão do modelo, sendo que em HFP o ajuste encontrado pode ser explicado em quase $85 \%$ dos casos, enquanto que para o consumo em HP o resultado obtido foi de 77\% de explicação. Gil-Vera (2017) também avaliou o $\mathrm{R}^{2}$, como medida para verificação da qualidade do ajuste do modelo para a série de demanda mensal de energia elétrica no Sistema Interconectado Nacional (SIN) da Colômbia, seu modelo obteve $\mathrm{R}^{2}$ mais siginificativo, próximo a $95 \%$, resultado este que é variável, de acordo com as características da série, como o período observado e a ocorrência ou não de tendência e sazonalidade.

Para os registros de MAPE, Lewis (1982) apud Puah et. al (2016, p. 274) desenvolveu uma escala para julgar a precisão do ajuste do modelo previsto usando o MAPE, como mostrado na Tabela 8.

Tabela 8 - Escala de julgamento da exatidão da previsão para MAPE.

\begin{tabular}{ll}
\hline MAPE & Precisão da previsão \\
\hline Menos do que $\mathbf{1 0 \%}$ & Altamente acurado \\
$\mathbf{1 1} \%$ a $\mathbf{2 0} \%$ & Boa previsão \\
$\mathbf{2 1 \%}$ a $\mathbf{5 0 \%}$ & Previsão razoável \\
$\mathbf{5 1 \%}$ ou mais & Previsão imprecisa \\
\hline
\end{tabular}

Fonte: Puah et. al. (2016, p. 274) 
Considerando os resultados obtidos pelo MAPE e a escala de julgamento da precisão da previsão do modelo, descrito na Tabela 9, proposta por Lewis, 1982 , verifica-se que os resultados encontrados variam entre altamente acurado à boa previsão, o que posiciona o ajuste como eficiente no que tange aos objetivos do trabalho, quanto à proposta para identificação de um modelo estatístico e a predição de valores futuros. Observa-se ainda, um comportamento similar, para a variável demanda HP e HFP, enquadrado como uma boa previsão. Sendo que a variável consumo apresenta uma maior diferença entre o HP e HFP, tendo sido a primeira enquadrada como boa previsão, e a segunda como uma previsão altamente acurada.

Verificou-se também o comportamento dos resíduos das séries, com o intuito de avaliar se estes são resíduos brancos, corroborando com a adequação do ajuste dos dados do modelo. Foi possível observar, que os resíduos não apresentam padrões óbvios para as séries analisadas de demanda e consumo HP e HFP, tendo sido verificada também por meio da matriz de correlação de Pearson, sendo os $p$-valores encontrados, para as variáveis não significativos ( $p$-valor $>0,05$ ), indicando assim que seus resíduos são aleatórios e o modelo aditivo de Holt-Winters se mostrou adequado para o ajuste das séries analisadas.

\subsection{PRECISÃO E VALIDAÇÃO DA PREVISÃO DOS MODELOS}

A fim de testar a precisão da previsão e como medida de validação dos resultados das séries de utilização de energia elétrica na UFU, para demanda e consumo em HP e HFP, usando o modelo aditivo de Holt-Winters, utilizou-se como base os registros mensais para as variáveis em questão no ano de 2018 para os meses de janeiro a agosto.

Para cada mês do ano de 2018 foi calculado os valores segundo os componentes do modelo, considerando o intervalo de confiança (95\%) e seus limites superior e inferior. A Figura 3 refere-se às séries de demanda HP, demanda HFP, consumo HP e consumo HFP e apresenta os valores previstos, 


\section{GESTÃO \& SUSTENTABILIDADE AMBIENTAL}

\section{sunisul sim}

intervalos de confiança (95\%), limites superior e inferior e o comportamento dos valores observados.

Figura 3 - Diagrama de validação do modelo para demanda HP, UFU/ campus Santa Mônica - 2018.

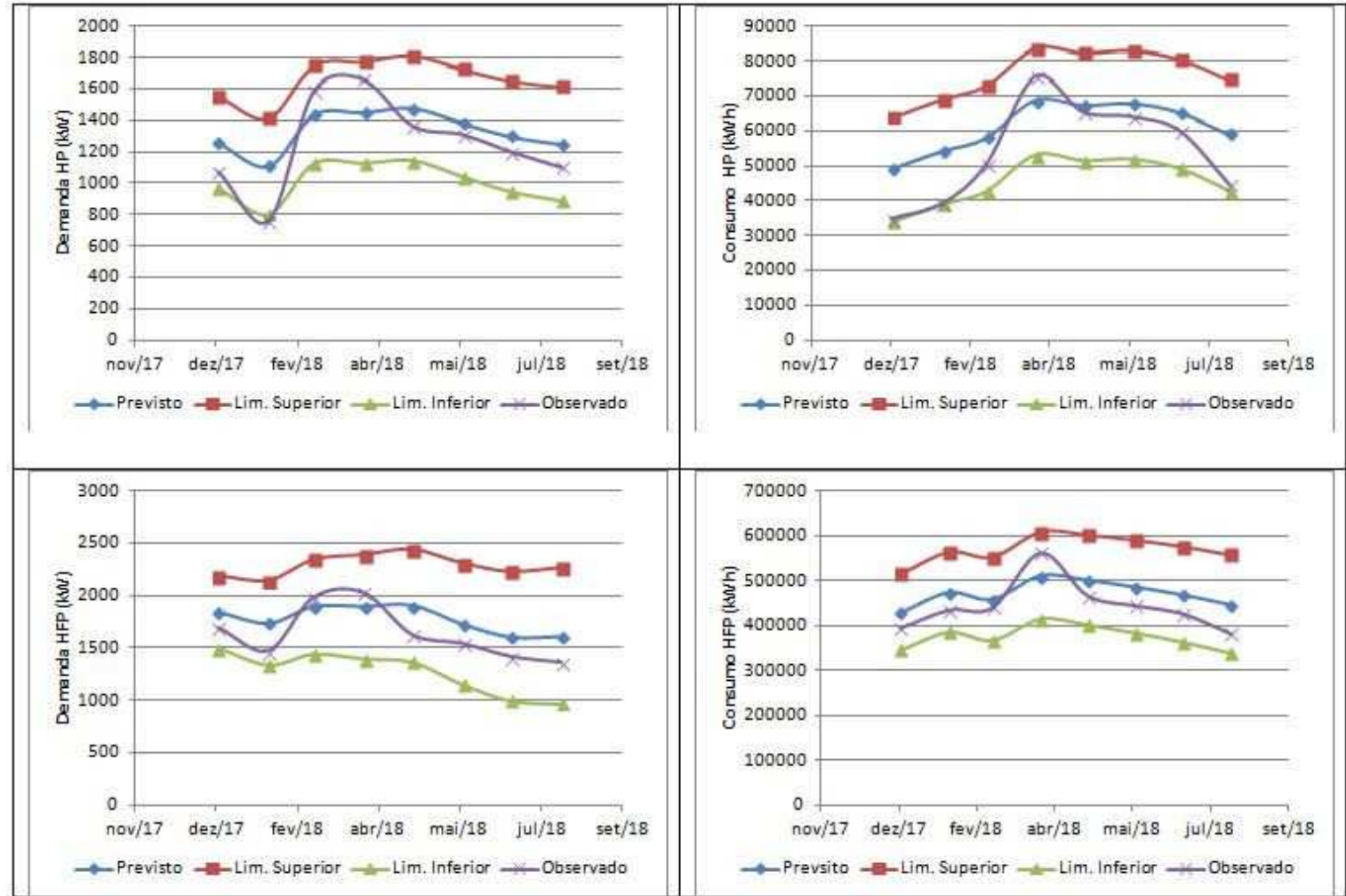

Fonte: Autores, 2018.

Deve-se ressaltar que o erro percentual médio, para as previsões em comparação com os valores observados, considerando os registros de janeiro a agosto de 2018, apresentou variação abaixo da média, respectivamente: i) 6,46\% para demanda HP; ii) 8,06\% para demanda HFP; iii) $12,11 \%$ para consumo HP e; iv) 6,54\% para consumo HFP. Essa característica, decrescente, do comportamento das previsões das variáveis está diretamente associada ao comportamento identificado para tendência nos registros dos anos de 2016 e 2017 (FIGURAS 1 e 2). Entretanto nota-se que o comportamento dos valores observados para cada uma das series analisadas, situam-se entre o intervalo de confiança e próximo aos valores previstos, validando assim, as previsões obtidas 


\section{GESTÃO \& SUSTENTABILIDADE} AMBIENTAL

\section{unisul sime}

e reforçando desta maneira a adequação do ajuste dos modelos identificados. Gil-Vera (2017), Silva et. al (2008) e Prado et. al (2014), utilizaram também desta metodologia para validação de suas previsões, tendo identificado comportamentos semelhantes aos observados na Figura 3.

\section{CONSIDERAÇÕES FINAIS}

Com base nas análises descritivas e ratificadas pelo Teste ANOVA, pôdese identificar características, presentes nas séries, de tendência e sazonalidade, sendo estas, diretamente relacionadas aos fatores estruturais, considerando o aumento do número de usuários e da área construída no campus Santa Mônica, para tendência, e seus calendários acadêmicos, considerando os períodos de férias e de plena atividade associados à variação sazonal.

O modelo com melhor ajuste para as séries foi o Aditivo de Holt-Winters, para todas as variáveis. Em relação às medidas de ajuste do modelo, destacase os resultados obtidos pelo $\mathrm{R}^{2}$ e MAPE posicionando o ajuste como eficiente no que tange aos objetivos do trabalho, para a identificação de um modelo estatístico. Deve-se ressaltar que comparativamente entre os modelos identificados, o ajuste que se mostrou mais adequado corresponde à série de consumo HFP e o menos adequado o modelo para consumo HP.

Os modelos de séries temporais permitiram a caracterização do comportamento da utilização da energia elétrica na UFU/ Santa Mônica, com base nas variáveis demanda de potência HP e HFP e consumo HP e HFP, além de apresentar o calculo da previsão das referidas variáveis, podendo ser replicado para os demais campi da UFU. A metodologia aplicada neste estudo caracteriza-se como ferramenta importante para a gestão da energia elétrica, melhorando o planejamento de sua utilização e consequentemente a redução de seus gastos, o que resulta na diminuição da utilização dos recursos ambientais necessários para a geração da energia elétrica. 


\section{GESTÃO \& SUSTENTABILIDADE} AMBIENTAL

\section{unisul}

\section{REFERÊNCIAS}

BRASIL. ANEEL - AGÊNCIA NACIONAL DE ENERGIA ELÉTRICA RESOLUÇÃO NORMATIVA № 414, DE 9 DE SETEMBRO DE 2010a. Disponível em: <http://www2.aneel.gov.br/cedoc/bren2010414.pdf>. Acesso em 14 de maio de 2018.

BRASIL. ANEEL - AGÊNCIA NACIONAL DE ENERGIA ELÉTRICA RESOLUÇÃO NORMATIVA № 418, DE 23 DE NOVEMBRO DE $2010 \mathrm{~b}$. Disponível em: <http://www2.aneel.gov.br/cedoc/ren2010418.pdf>. Aceso em 14 de maio de 2018.

BRASIL. PRESIDÊNCIA DA REPÚBLICA. DECRETO № 6.096, DE 24 DE ABRIL DE 2007.

BRILLINGER, D. R. Time Series: General, In International Encyclopedia of the Social \& Behavioral Sciences (Second Edition), edited by James D. Wright,, Elsevier, Oxford, 2015, Pages 341-346, ISBN 9780080970875, Disponível em: $<$ https://www.sciencedirect.com/science/article/pii/B9780080970868420842>.

Acesso em: 21 de maio de 2018.

CONARQ - Arquivo Nacional, Classificação, Temporalidade e Destinação de Documentos de Arquivos Relativos às Atividades- Meio da Administração Pública, p. 71, 2001.

CAPES - COORDENAÇÃO DE APERFEIÇOAMENTO DE PESSOAL DE NíVEL SUPERIOR, Número de estudantes de pós-graduação (mestrado e doutorado), 2017. Disponíveis em:

$<$ https://sucupira.capes.gov.br/sucupira/public/consultas/coleta/discente/listaDis cente.jsf>. Acesso em 01 de maio de 2018.

DINCER, I. Renewable energy and sustainable development: a crucial review, Renewable and Sustainable Energy Reviews, Volume 4, Issue 2, 2000, Pages 157-175, ISSN 1364-0321. Disponível em <http://www.sciencedirect.com/science/article/pii/S1364032199000118>.

Acesso em 15 de maio de 2018.

EMPRESA DE PESQUISA ENERGÉTICA - EPE, MINISTÉRIO DE MINAS E ENERGIA - MME, Brasil, Anuário Estatístico de Energia Elétrica. Brasil, 2017.

FERRAZ, M, I, F. Uso de modelos de séries temporais na previsão da série de precipitação pluviais mensais no município de Lavras - MG. 97p. Dissertação (Mestrado em Estatística e Experimentação Agropecuária) Universidade Federal de Lavras, Lavras, 1999.

GIL-VERA, V. Pronóstico de la demanda mensual de electricidad con series de tiempo. Revista EIA, [S.I], v, 13, n. 26, p. 111-120, mar. 2017. ISSN 2463-0950. Disponível

em: $<$ HTTPS://revsitas.eia.edu.co/index.php/reveia/article/view/749>. Acesso em : 26 de abril de 2018. 


\section{GESTÃO \& SUSTENTABILIDADE AMBIENTAL \\ sunisul}

GOLDEMBERG, J. O estado atual do setor elétrico brasileiro. Revista USP, v. 1, p. 39-44, 2015.

KHALIQ, A., BATOOL, S. A., CHAUDHRY, M. N., Seasonality and trend analysis of tuberculosis in Lahore, Pakistan from 2006 to 2013, Journal of Epidemiology and Global Health, Volume 5, Issue 4, Pages 397-403, 2015, ISSN 2210-6006. Disponível

em:

<http://www.sciencedirect.com/science/article/pii/S221060061500088X>.

Acesso em: 08 de agosto de 2018.

LO, K., Energy conservation in China's higher education institutions, Energy Policy, Volume 56, 2013, Pages 703-710, ISSN 0301-4215. Disponível em: <http://www.sciencedirect.com/science/article/pii/S0301421513000621>.

Acesso em 15 de maio de 2018

MENKES, M. , Instrumentos econômicos aplicados em programas de eficiência energética. In: IV Encontro Nacional da ECOECO. Belém, 2001. p. 1-26. 2003. Disponível em: HTTP://www.ecoeco.org.br/conteudo/publicacoes/encontros/iv_en/mesa3/1.pdf. Acesso em 20 de abril de 2018.

MORETTIN, P. A., TOLOI, C. M. C. Análise de séries temporais, $2^{a}$ edição ver. Ampli., São Paulo, Blucher, 2006.

OLIVEIRA, L. S.,. Gestão do consumo de energia elétrica no campus da UnB. Dissertação de Mestrado em Engenharia Elétrica, Publicação PPGENE.DM-268/06, Departamento de Engenharia Elétrica, Universidade de Brasília, DF, 219 p, 2006.

PEREIRA FILHO, A. J., Análise da escassez hídrica brasileira em 2014. Revista USP, p. 125, 2015.

PRIETO, E. C., Os desafios institucionais e municipais para implantação de uma cidade universitária: o Campus Glória da Universidade Federal de Uberlândia. 268 f. Dissertação (Mestrado em Geografia) - Universidade Federal de Uberlândia, Uberlândia, 2005.

PRIETO, E. C., Universidade Sustentável: desafios e compromissos da educação e da gestão ambiental na Universidade Federal de Uberlândia, MG. 173 f. Tese (Doutorado em Geografia) - Universidade Federal de Uberlândia, Uberlândia, 2012.

PRADO, J. R.; SAFADI, T. ; SILVA, J. P. . Modelagem de séries temporais de demanda de energia elétrica da Universidade Federal de Lavras, correspondentes ao período de 1995 a 2013. Revista da Estatística da Universidade Federal de Ouro Preto, v. 3, p. 564, 2014.

PROPLAD - PRÓ-REITORIA DE PLANEJAMENTO E ADMINISTRAÇÃO, Dados Gerais 2016 Ano Base 2015. Uberlândia, MG. 2016. 247 p. Universidade Federal de Uberlândia (UFU). Disponível em: <http://www.proplad.ufu.br/sites/proplad.ufu.br/files/media/arquivo/anuario_201 6_-_base_2015.pdf>. Acesso em: 27 de setembro de 2017. 


\section{GESTÃO \& SUSTENTABILIDADE AMBIENTAL \\ ¿unisul}

PROPLAD - PRÓ-REITORIA DE PLANEJAMENTO E ADMINISTRAÇÃO, Dados Gerais 2017 Ano Base 2016. Uberlândia, MG. 2017. 35 p. Universidade Federal de Uberlândia (UFU). Disponível em: <http://www.proplad.ufu.br/sites/proplad.ufu.br/files/media/arquivo/folder_2017_ano-base_2016.pdf>. Acesso em: 13 de agosto de 2018.

PROPLAD - PRÓ-REITORIA DE PLANEJAMENTO E ADMINISTRAÇÃO, Dados Gerais 2018 Ano Base 2017. Uberlândia, MG. 2018. 12 p. Universidade Federal de Uberlândia (UFU). Disponível em: <http://www.proplad.ufu.br/sites/proplad.ufu.br/files/media/arquivo/dados_gerais 2018_-_ano_base_2017.pdf>. Acesso em: 12 de setembro de 2018.

PUAH, Y.J, HUANG, Y. F., CHUA, K.C., LEE, T.S., River catchment rainfall series analysis using additive Holt-Winters method.

Journal of Earth System Science, 2016, Vol.125(2), p.269(15). Springer India. Disponível em: <https://doi-org.ez34.periodicos.capes.gov.br/10.1007/s12040016-0661-6>. Acesso em: 27 de junho de 2018.

SILVA, C. O. S.; NASSAR, C. A. G. Análise do uso da energia elétrica no Instituto Federal Fluminense campus Campos Guarus. Revista de Gestão Ambiental e Sustentabilidade - GeAS. Vol. 5, N.3. 2016.

SILVA, M. I. S; GUIMARÃES, E. C; TAVARES, M., Previsão da temperatura média mensal de Uberlândia, MG, com modelos de séries temporais. Revista Brasileira de Engenharia Agrícola e Ambiental. Vol.12, n.5, p.480-485, 2008.

SERNA, M.S; CARVALHO, C.S; MENEZES, R.F; TEODORO, J.R; OLIVEIRA, L.M. Eficiência Energética Na Gestão Da Conta De Energia Elétrica Da Universidade Federal De Sergipe. In: XI Colóquio Internacional sobre Gestão Universitária na América do Sul e II Congresso Internacional IGLU. Florianópolis, SC. 2011. 